\title{
A Novel Quadrature Signal Estimation Method for a Planar Capacitive Incremental Displacement Sensor
}

\author{
Jian-Ping $\mathrm{Yu}^{1}$, Wen Wang ${ }^{2}$, Xin $\mathrm{Li}^{1}$, Zhao-Zhong Zhou ${ }^{1}$ \\ ${ }^{1}$ College of Mechanical Engineering, Quzhou University, Jiuhua Rd., No. 78, 324000, Quzhou, China, \\ yujianping@zju.edu.cn \\ ${ }^{2}$ School of Mechanical Engineering, Hangzhou Dianzi University, Baiyang 2nd Rd., No. 1158, 310018, Hangzhou, China
}

\begin{abstract}
This paper presents a novel phase-shift arctangent (PSA) interpolation method to improve the measurement accuracy of a planar capacitive incremental displacement sensor. Signals of planar capacitive micro-sensors acquire waveform errors, including sensitivity differences and phase-shift errors, because of static errors and dynamic disturbances. In the proposed PSA scheme, such errors are removed completely by loading a particular arctangent function. Moreover, measuring efficiency of the proposed planar capacitive sensors is improved by combining coarse measurement and fine estimation. Experiments show unanimous results to model-based fitting. When electrode length is four times the gap distance, applying the PSA interpolation method decreases waveform errors from more than $4 \%$ to $1.72 \%$.
\end{abstract}

Keywords: Phase-shift arctangent method, capacitive displacement sensor, static and dynamic errors, accuracy improvement.

\section{INTRODUCTION}

In the past few decades, planar capacitive incremental displacement sensor (PCIDS) has been proposed for use in large-scale micro/nanoprecision measurement applications, such as fabrication of optical devices and semiconductors, cell manipulation, and portable probe storage systems [1][6]. Similar to most encoder-like prototypes, PCIDS offers advantages such as large measurement scale, high sensitivity, fast dynamic response, effective cost, and reliable stability. However, the main shortcomings of PCIDS are some inevitable effects, such as static errors (mainly as alignment errors) and dynamic disturbances (including kinematic errors and vibration disturbances) on signal waveform errors [7]-[10].

Influences of static errors and dynamic disturbances are usually in the form of sensitivity differences and phase- shift errors. Conventional solutions to reduce them can be classified into system calibrations and structural improvements. Kuijipers et al. connected two comb actuators along the slide path of a moving plate to guarantee its mobility. Accurate measurement of PCIDS depends closely on the correct installation of the two comb actuators [4]. Kim et al. reduced gap distance instability by assembling two sensor substrates that face each other after coating them with $0.4 \mu \mathrm{m}$ diamond-like carbon film and using two look-up tables to linearize the measured capacitances to displacements [5]. Huang et al. used rigid mechanical spacers to control gap distance. However, this approach eliminates the advantages of non-contact measurement [6]. Heydemann analyzed primary errors of co-sinusoidal signals of incremental optical sensors. Least squares fitting was adopted to compensate for these errors [10].

Displacement information is contained in the phase and amplitude of periodic waveforms of PCIDS. Commonly, PCIDS signals are squared into electrical pulses, the number of which is counted to determine displacement information [11]-[15]. Tan et al. introduced an interpolation method based on constructing and squaring a set of high-order sinusoids. A look-up table was used offline to compensate for interpolation errors [11]. Hu et al. proposed a method to solve $\lambda / 16$ bidirectional subdivision for quadrature signals by constructing two sets of reference signals [12]. However, these methods would easily neglect displacement information in a single period. To improve measurement resolution, Benammar et al. proposed a multiple phaseshifted sinusoid method, where displacement information in a signal period is also calculated [13]. Nevertheless, given that sinusoidal waveform is not completely linear, the theoretical absolute error of this scheme is $0.028^{\circ}$ within $360^{\circ}$.

This paper presents a novel interpolation method that can reduce the effect of static errors and dynamic disturbances effectively while improving PCIDS measurement resolution. The mathematical sensor model against harmonic disturbances is presented in Sec. 2. A phase-shift arctangent (PSA) interpolation method is proposed in Sec. 3. The experimental results and summary are presented in Sec. 4 and Sec. 5, respectively. 


\section{SENSOR MODEL AGAINST HARMONIC DISTURBANCES}

\section{A. Sensor mechanism}

Fig.1. shows the schematic structure of the proposed PCIDS. The sensor consists of a fixed plate (FP) and a moving plate (MP). In the figure, $P$ is the electrode interval on MP, $l$ is the electrode length of MP and FP, $g$ is the gap distance of MP and FP, and $L_{S}$ is the vertical distance of sensing electrodes to $X$ or $Y$ axis. Common electrodes on MP are parallel and electrically connected, on which a sinusoidal voltage $V_{\text {input }}$ should be applied during measurement. Eight sensing electrodes on FP are positioned symmetrically around the rotational center $O$ (an assumed origin). The sensing electrodes and common electrodes consist of eight sensing capacitors, namely, $\mathrm{SX}_{1 \mathrm{~N}}, \mathrm{SX}_{1 \mathrm{Q}}$, $\mathrm{SX}_{2 \mathrm{~N}}, \mathrm{SX}_{2 \mathrm{Q}}, \mathrm{SY}_{2 \mathrm{~N}}, \mathrm{SY}_{2 \mathrm{Q}}, \mathrm{SY}_{1 \mathrm{~N}}$, and $\mathrm{SY}_{1 \mathrm{Q}}$.

Sensing capacitors $\mathrm{SX}_{1 \mathrm{~N}}, \mathrm{SX}_{1 \mathrm{Q}}, \mathrm{SX}_{2 \mathrm{~N}}$, and $\mathrm{SX}_{2 \mathrm{Q}}$ provide $X$ direction displacement information. $\mathrm{SY}_{1 \mathrm{~N}}, \mathrm{SY}_{1 \mathrm{Q}}, \mathrm{SY}_{2 \mathrm{~N}}$, and $\mathrm{SY}_{2 \mathrm{Q}}$ provide $Y$ direction displacement information. Four sensing capacitors $\left(\mathrm{SX}_{1 \mathrm{Q}}, \mathrm{SX}_{2 \mathrm{Q}}, \mathrm{SY}_{1 \mathrm{Q}}, \mathrm{SY}_{2 \mathrm{Q}}\right)$ are all (1/4)P shifted to sensing capacitors $\left(\mathrm{SX}_{1 \mathrm{~N}}, \mathrm{SX}_{2 \mathrm{~N}}, \mathrm{SY}_{1 \mathrm{~N}}, \mathrm{SY}_{2 \mathrm{~N}}\right)$ in their respective measurement directions. Signals from $S_{1 N}$, $\mathrm{SX}_{2 \mathrm{~N}}, \mathrm{SY}_{1 \mathrm{~N}}$, and $\mathrm{SY}_{2 \mathrm{~N}}$ are implied to have a $(1 / 2) \pi$ phase shift to signals $\mathrm{SX}_{1 \mathrm{Q}}, \mathrm{SX}_{2 \mathrm{Q}}, \mathrm{SY}_{1 \mathrm{Q}}$, and $\mathrm{SY}_{2 \mathrm{Q}}[16]$.

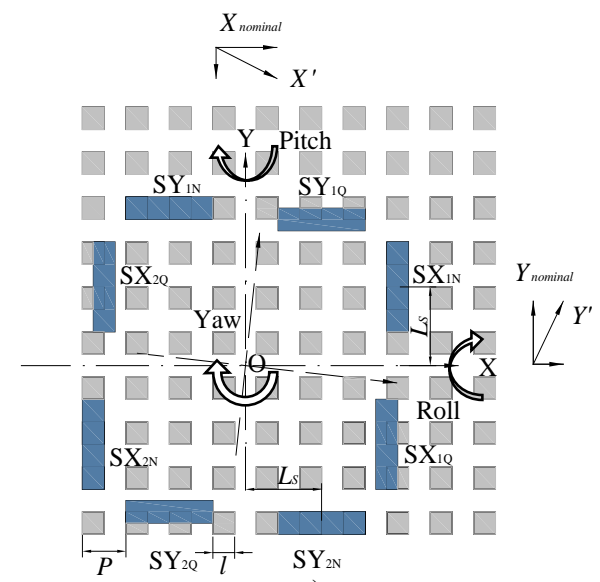

a)

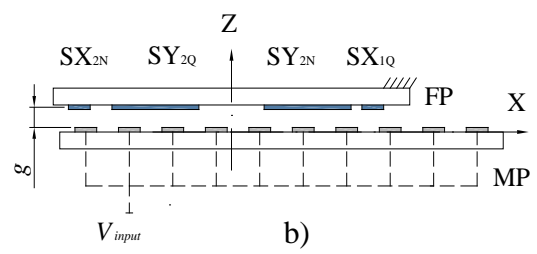

Fig.1. Schematic view of a PCIDS: a) front view; b) cross view.

\section{B. Signal model against disturbances}

Setting aside fringe effects, signals from eight sensing capacitors are all in perfect periodic triangular waveforms given that common electrodes on MP are parallel. However, in reality, fringe effects would introduce severe uncertain distortion (rounded) on signal waveforms. To clarify the influence of fringe effects on signal nonlinearity, a full capacitance model is calculated under Maxwell's equations first.
As illustrated in Fig.1., common electrodes are periodically positioned on MP and driven by a positive voltage $V_{\text {input }}$. Meanwhile, sensing electrodes on FP are virtually ground to diminish electrical interferences. Margins on MP and FP are both ground. If the potential on the FP and MP is assumed as $\Phi_{1}(x)$ and $\Phi_{2}(x)$, respectively, then its Fourier expansion can be expressed as:

$$
\begin{gathered}
\Phi_{1}(x)=0 \\
\Phi_{2}(x)=\frac{V_{\text {input }}}{2}+\sum_{n=\text { odd }} \frac{2 V_{\text {input }}}{n \pi} \sin \left(k_{n} x\right)
\end{gathered}
$$

where $k_{n}=\frac{n \pi}{l}$.

When MP has a travel position $\delta$ in its direction, its charge distribution on the surface of FP can be determined by Maxwell's equation.

$$
\sigma=-\varepsilon_{0} \varepsilon_{r}\left[\frac{V_{\text {input }}}{2 g}+\sum_{n=\text { odd }} \frac{2 V_{\text {input }}}{l} \frac{\sin \left(k_{n}(x-\delta)\right)}{\sinh \left(k_{n} g\right)}\right]
$$

Given that MP is grounded in the period [-l, 0], we are interested only in the charge in the period $[0,1]$. Integrating the charge distribution on FP can achieve the capacitance as:

$$
C(x)=\frac{Q}{V_{\text {input }}}=-\varepsilon_{0} \varepsilon_{r} l\left[\frac{l}{2 g}-\sum_{n=\text { odd }} \frac{4}{k_{n} l \sinh \left(k_{n} g\right)} \cos \left(k_{n} x\right)\right]
$$

As in (4), electrode length is implied to be a key factor that determines sensor waveform nonlinearity to gap-distance ratio $(\mathrm{l} / \mathrm{g})$. Fig.2. illustrates a standard deviation comparison of waveform deformation errors of the proposed sensor signals to ideal triangular and sinusoidal waveforms under different $l / g$ ratio. The comparison indicates that the actual waveform is more closely dependent on sinusoidal waveforms than triangular waveforms, especially when $l / g$ ratio is less than two. Thus, in the actual PCIDS model, signals can be considered sinusoidal waveforms. Moreover, when a common arctangent operation is applied, standard deviation of waveform deformation errors can still be decreased.

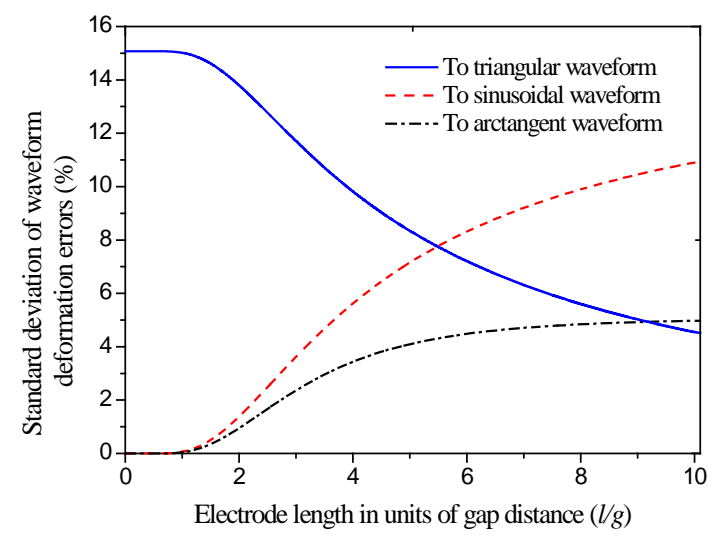

Fig.2. Standard deviation comparison of waveform deformation errors under different $l / g$ ratio. 
On the basis of this conclusion, when MP and FP are completely parallel and facing each other as shown in Fig.1., normalized nominal output signals from the eight sensing capacitors can be expressed as:

$$
\begin{aligned}
& \left\{\begin{array}{l}
X_{1 N}^{\text {nominal }}=X_{2 N}^{\text {nominal }}=\cos \left[2 \pi \cdot\left(X_{\text {nominal }}\right) / P\right] \\
-X_{1 Q}^{\text {nominal }}=X_{2 Q}^{\text {nominal }}=\sin \left[2 \pi \cdot\left(X_{\text {nominal }}\right) / P\right]
\end{array}\right. \\
& \left\{\begin{array}{l}
Y_{1 N}^{\text {nominal }}=Y_{2 N}^{\text {nominal }}=\cos \left[2 \pi \cdot\left(Y_{\text {nominal }}\right) / P\right] \\
-Y_{1 Q}^{\text {nominal }}=Y_{2 Q}^{\text {nominal }}=\sin \left[2 \pi \cdot\left(Y_{\text {nominal }}\right) / P\right]
\end{array}\right.
\end{aligned}
$$

where $X_{\text {nominal }}$ and $Y_{\text {nominal }}$ represent the theoretical displacement of the MP in $X$ and $Y$ direction.

However, excluding fringe effects, static errors and dynamic disturbances are inevitable during measurement, thereby affecting PCIDS in the form of roll, yaw, pitch, and vertical movements of MP and FP.

Those static and dynamic disturbances are usually minimal. Yaw movement can be seen as a combination of two separate linear movements in the $X$ and $Y$ directions. Linear movements of $\mathrm{SX}_{1 \mathrm{~N}}, \mathrm{SX}_{1 \mathrm{Q}}, \mathrm{SX}_{2 \mathrm{~N}}$, and $\mathrm{SX}_{2 \mathrm{Q}}$ in the $X$ direction cause each to have a phase-shift error as shown in (7). Similarly, linear movements of $\mathrm{SY}_{1 \mathrm{~N}}, \mathrm{SY}_{1 \mathrm{Q}}, \mathrm{SY}_{2 \mathrm{~N}}$, and $\mathrm{SY}_{2 \mathrm{Q}}$ in the $Y$ direction cause each to have a phase-shift error as shown in (8).

$$
\begin{gathered}
\left\{\begin{array}{l}
X_{1 N}^{\prime}=\cos \left[2 \pi \cdot\left(X_{\text {nominal }}+X_{\theta z}\right) / P\right] \\
X_{1 Q}^{\prime}=-\sin \left[2 \pi \cdot\left(X_{\text {nominal }}-X_{\theta z}\right) / P\right] \\
X^{\prime}{ }_{2 N}=\cos \left[2 \pi \cdot\left(X_{\text {nominal }}-X_{\theta z}\right) / P\right] \\
X^{\prime}{ }_{2 Q}=\sin \left[2 \pi \cdot\left(X_{\text {nominal }}+X_{\theta z}\right) / P\right]
\end{array}\right. \\
\left\{\begin{array}{l}
Y_{1 N}^{\prime}=\cos \left[2 \pi \cdot\left(Y_{\text {nominal }}+Y_{\theta z}\right) / P\right] \\
Y_{1 Q}^{\prime}=-\sin \left[2 \pi \cdot\left(Y_{\text {nominal }}-Y_{\theta z}\right) / P\right] \\
Y^{\prime}{ }_{2 N}=\cos \left[2 \pi \cdot\left(Y_{\text {nominal }}-Y_{\theta z}\right) / P\right] \\
Y^{\prime}{ }_{2 Q}=\sin \left[2 \pi \cdot\left(Y_{\text {nominal }}+Y_{\theta z}\right) / P\right]
\end{array}\right. \\
X_{\theta z}=Y_{\theta z}=L_{S} \cdot \sin \left(\theta_{z}\right)
\end{gathered}
$$

where $\theta_{Z}$ is the yaw angle of MP; and $X_{\theta Z}$ and $Y_{\theta Z}$ are the linear movements of MP to the eight sensing electrodes on FP caused by yaw angle. PCIDS in Fig.1. is symmetrically designed. Thus, $X_{\theta Z}$ is equivalent to $Y_{\theta Z}$.

Movements caused by roll and pitch angles can also be considered a combination of linear movement in the $Z$ direction and linear movement in the $X$ or $Y$ direction. However, linear movements in the $X$ or $Y$ direction in this study are neglected because they are too small compared with the linear movement in the $Z$ direction. Thus, influences of roll and pitch movement are considered the same as vertical movements, affecting only the amplitude of PCIDS outputs. Then, the actual PCIDS model against static and dynamic disturbances is illustrated as (10), (11).

$$
\begin{array}{r}
\left\{\begin{array}{l}
X_{1 N}=K_{X 1 N} \cdot \cos \left[2 \pi \cdot\left(X_{\text {nominal }}+X_{\theta z}\right) / P\right] \\
X_{1 Q}=-K_{X 1 Q} \cdot \sin \left[2 \pi \cdot\left(X_{\text {nominal }}-X_{\theta z}\right) / P\right] \\
X_{2 N}=K_{X 2 N} \cdot \cos \left[2 \pi \cdot\left(X_{\text {nominal }}-X_{\theta z}\right) / P\right] \\
X_{2 Q}=K_{X 2 Q} \cdot \sin \left[2 \pi \cdot\left(X_{\text {nominal }}+X_{\theta z}\right) / P\right]
\end{array}\right. \\
\left\{\begin{array}{l}
Y_{1 N}=K_{Y 1 N} \cdot \cos \left[2 \pi \cdot\left(Y_{\text {nominal }}+Y_{\theta z}\right) / P\right] \\
Y_{1 Q}=-K_{Y 1 Q} \cdot \sin \left[2 \pi \cdot\left(Y_{\text {nominal }}-Y_{\theta z}\right) / P\right] \\
Y_{2 N}=K_{Y 2 N} \cdot \cos \left[2 \pi \cdot\left(Y_{\text {nominal }}-Y_{\theta z}\right) / P\right] \\
Y_{2 Q}=K_{Y 2 Q} \cdot \sin \left[2 \pi \cdot\left(Y_{\text {nominal }}+Y_{\theta z}\right) / P\right]
\end{array}\right.
\end{array}
$$

where $K_{X 1 N}, K_{X 1 Q}, K_{X 2 N}, K_{X 2 Q}, K_{Y 1 N}, K_{Y 1 Q}, K_{Y 2 N}, K_{Y 2 Q}$ represent approximate relationships of sensor sensitivity to roll, pitch angles and vertical movement of the MP.

\section{PRINCIPLE OF PSA INTERPOLATION METHOD}

The above analysis indicates that PCIDS accuracy is closely dependent on that of acquired waveforms. Thus, reducing effects of static and dynamic disturbances is of paramount importance. Normalizing (10) and (11) would remove the effect of sensitivity differences, turning signals into $X^{\prime}{ }_{1 N}, X_{1 Q}, X_{2 N}, X^{\prime}{ }_{2 Q}, Y_{1 N}, Y_{1 Q}, Y_{2 N}{ }_{2 N}$, and $Y^{\prime}{ }_{2 Q}$ as shown in (7) and (8). However, if arctangent waveforms are then directly derived from normalized signals as shown in (12) and (13), then phase-shift errors would have unexpected influences on displacement estimation.

$$
\begin{aligned}
& \left\{\begin{array}{l}
X_{1}=\frac{P}{2 \pi} \arctan \frac{-X_{1 Q}^{\prime}}{X_{1 N}^{\prime}} \\
X_{2}=\frac{P}{2 \pi} \arctan \frac{X_{2 Q}^{\prime}}{X_{2 N}^{\prime}}
\end{array}\right. \\
& \left\{\begin{array}{l}
Y_{1}=\frac{P}{2 \pi} \arctan \frac{-Y^{\prime}{ }_{1 Q}}{Y_{1 N}^{\prime}} \\
Y_{2}=\frac{P}{2 \pi} \arctan \frac{Y_{2 Q}^{\prime}}{Y_{2 N}^{\prime}}
\end{array}\right.
\end{aligned}
$$

In the PSA scheme, the $X-Y$ displacement signals are obtained by a particular arctangent function as shown in (14) and (15). Effects of phase-shift errors on $X-Y$ quadrature waveforms are also removed. The following expressions contain only information of the theoretical $X-Y$ movements as $X_{\text {nominal }}$ and $Y_{\text {nominal }}$.

$$
\begin{gathered}
X_{\text {nominal }}=\frac{P}{2 \pi} \arctan \frac{-X_{1 Q}^{\prime}+X_{2 Q}^{\prime}}{X_{1 N}^{\prime}+X^{\prime}{ }_{2 N}} \\
Y_{\text {nominal }}=\frac{P}{2 \pi} \arctan \frac{-Y_{1 Q}^{\prime}+Y_{2 Q}^{\prime}}{Y_{1 N}^{\prime}+Y^{\prime}{ }_{2 N}}
\end{gathered}
$$

Furthermore, after acquiring signal models as shown in (14) and (15), the PSA method improves PCIDS measurement resolution by combining coarse displacement measurement and fine displacement estimation value as (16). 


$$
D=D_{C}+D_{F}
$$

Fig.3. illustrates the basic principle of the PSA interpolation method. First, $N$ sets of shifted arctangent signals are derived from (17). Then, certain segments of each shifted arctangent are combined to form a useful triangular waveform for later interpolation.

$$
T(\theta-i \varphi)=\frac{P}{2 \pi} \arctan \left[\frac{\tan (\theta)-\tan (i \varphi)}{1+\tan (\theta) \cdot \tan (i \varphi)}\right]
$$

where $i=1,2,3, \ldots \ldots, 2 N-1, \varphi=\pi /(2 N), \theta=2 \pi X_{\text {nominal }} / P$ or $2 \pi Y_{\text {nominal }} / P$.

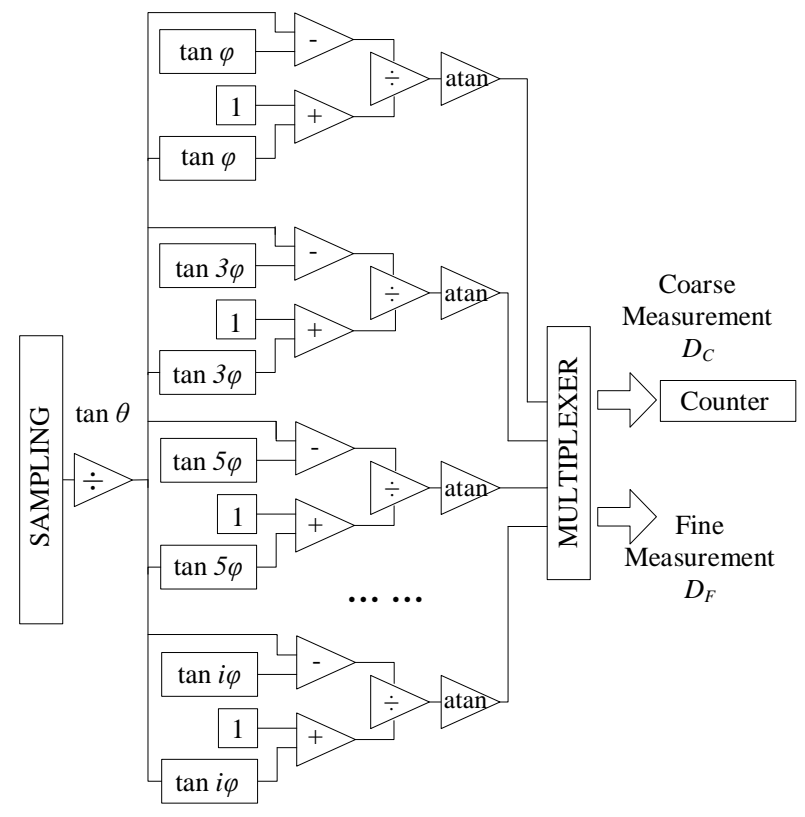

Fig.3. Principle of PSA interpolation method.

PSA scheme (17) shifts the phase value of the given signals only and not on the specific value of signal magnitudes. Thus, when given signals have nonlinearity errors, the number of modulation $N$ neither enhances nor rejects nonlinearity errors introduced by static and dynamic errors.

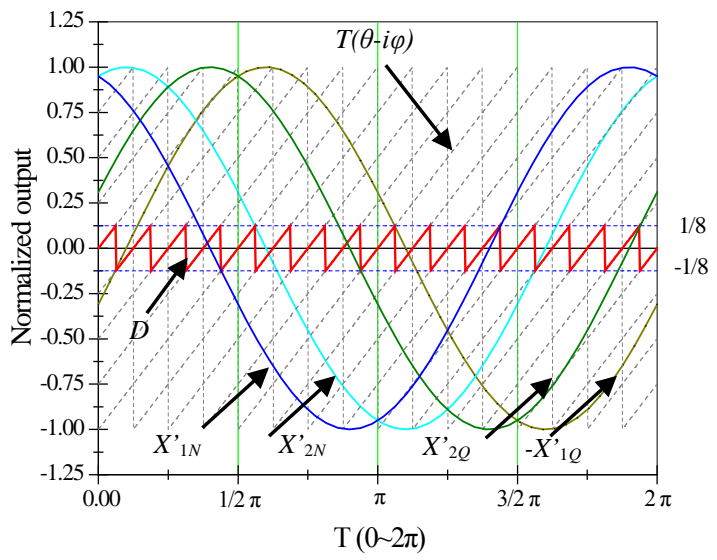

Fig. 4. Simulation results of a PSA interpolation method when $N=8$.
The generated triangular signal is first squared into pulses. Coarse displacement of MP $D_{C}$ can be measured by counting the number of pulses. The fine displacement $D_{F}$ is then determined by the analog output in the last single period.

Analog signal measurement is retained in the PSA interpolation method. Thus, lost displacement information in the conventional interpolation method is now obtained by the PSA interpolation method.

Fig.4. presents the simulation results of the PSA interpolation method when $N=8$. (14) completely removes influences of phase-shift errors and sensitivity differences. Theoretical $X$ movement as $X_{\text {nominal }}$ is acquired. The phaseshift function (17) generates a set of triangular waveforms. PCIDS measurement resolution and efficiency are improved through calculation (16).

Conclusively, the following points are the advantages of the PSA interpolation method over traditional phase-shift interpolation methods: 1) effects of static errors and dynamic disturbances are theoretically removed; 2) arctangent waveform is absolutely linear, and PSA signals do not have theoretical nonlinearity errors; 3) PSA signals are derived from a set of sinusoidal and cosine signals; waveform deformation errors are well balanced within a full period; 4) PSA interpolation acquires higher measurement resolution by combining coarse measurement and fine estimation; the improvement is that displacement information in the final single period is calculated in the fine estimation.

\section{EXPERIMENTS AND DISCUSSION}

A PCIDS system is constructed as shown in Fig.5. and consists of a micro motion stage with nanometer resolution, a vertical motion stage, two tilting motion stages, a rotational motion stage, PCIDS, and a PSA interpolation system. MP and FP are fabricated as a printed circuit board. NANOMOTION ${ }^{\circledR}$ motor with a resolution of $10 \mathrm{~nm}$ is applied to provide the displacement of the micro motion stage. Tilting and rotating stages are mounted for adjusting installation errors and system calibration. The vertical motion stage provides $Z$ direction motion for gap distance adjustment. Phase-locked detection is chosen for signal sampling, and noise errors would be removed effectively. The PSA interpolation system, including phase-shift, counting, and interpolation, is constructed on LABVIEW ${ }^{\circledR}$.

Reasonable system calibration should be carried out to protect PCIDS measurement from the influences of static errors. However, dynamic disturbances mainly in the form of kinematic errors and vibration disturbances would bring unexpected errors during measurement. Even sophisticated calibration cannot completely remove static errors. Thus, in most circumstances, static errors and dynamic disturbances are inevitable in PCIDS measurement.

On the basis of the principle of PSA interpolation method in Sec. 3, the PCIDS developed in this study can prevent the effects of measurement disturbances, such as roll, yaw, pitch, and vertical errors. Thus, if experiments reveal unanimous mathematical calculation results, then the ability of PSA interpolation method to ensure the satisfactory performance of PCIDS despite measurement disturbances can be validated. 


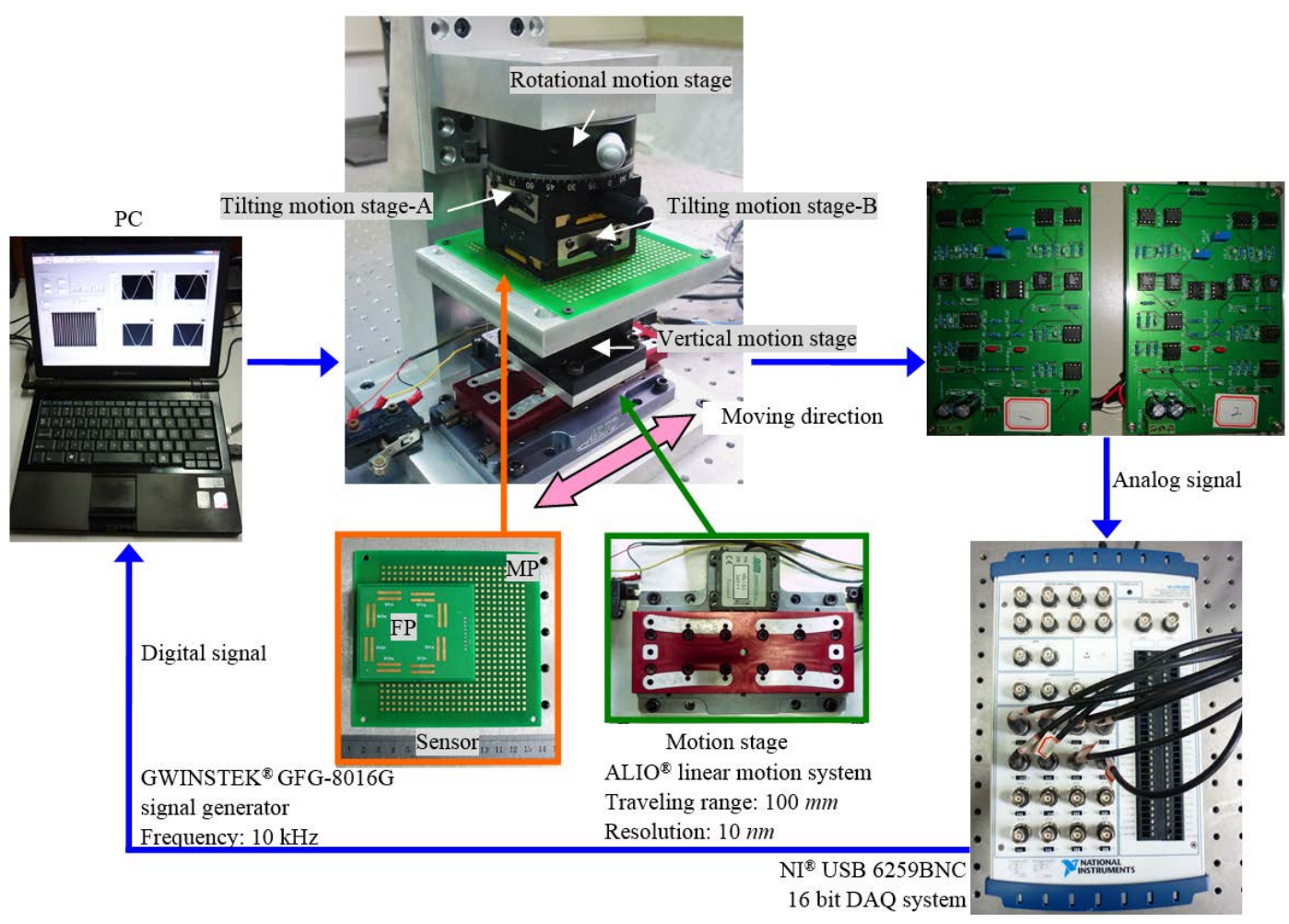

Fig.5. Test bench of the proposed planar capacitive incremental displacement sensor system.

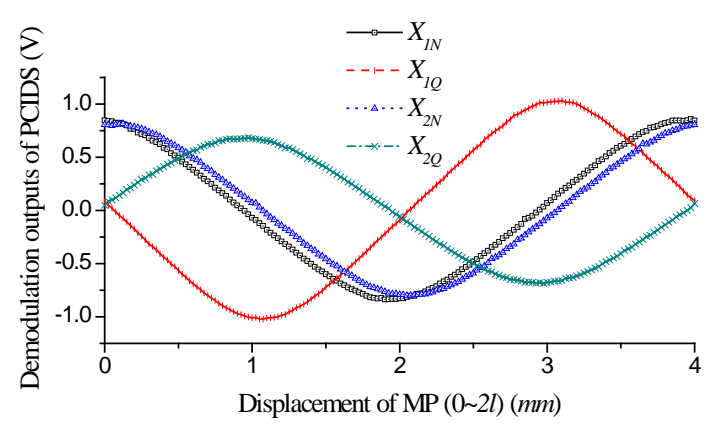

a)

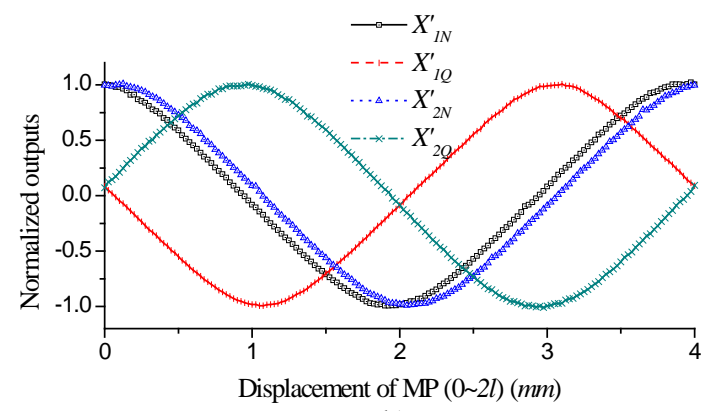

b)

Fig.6. PCIDS signals in a single period when $l / g=4$ :

a) demodulation outputs of PCIDS as $X_{1 N}, X_{2 N}, X_{1 Q}, X_{2 Q}$;

b) normalized waveforms as $X^{\prime}{ }_{1 N}, X^{\prime}{ }_{2 N}, X^{\prime}{ }_{1 Q}, X^{\prime}{ }_{2 Q}$.

Fig.6.a) depicts the demodulation outputs of PCIDS as $X_{1 N}$, $X_{2 N}, X_{1 Q}$, and $X_{2 Q}$ in a single period when the electrode length is four times the gap distance $(l / g=4)$. Even after system calibration, static errors and dynamic disturbances are not completely removed, thereby introducing severe sensitivity differences and phase-shift errors to output waveforms.

Fig.6.b) depicts normalized waveforms of $X^{\prime}{ }_{1 N}, X^{\prime}{ }_{2 N}, X^{\prime}{ }_{1 Q}$, and $X^{\prime}{ }_{2 Q}$, with sensitivity differences removed. However, phase-shift errors remain. If acquired normalized waveforms are considered ideal quadrature waveforms, then phase-shift errors would diminish measurement accuracy. Fig.7. plots the arctangent waveform directly from normalized waveforms (12). Acquired waveforms exhibit severe distortion. Fig.8. plots waveform errors and shows that the standard deviations of the $X_{1}$ and $X_{2}$ waveform errors are as high as $4.43 \%$ and $4.37 \%$, respectively.

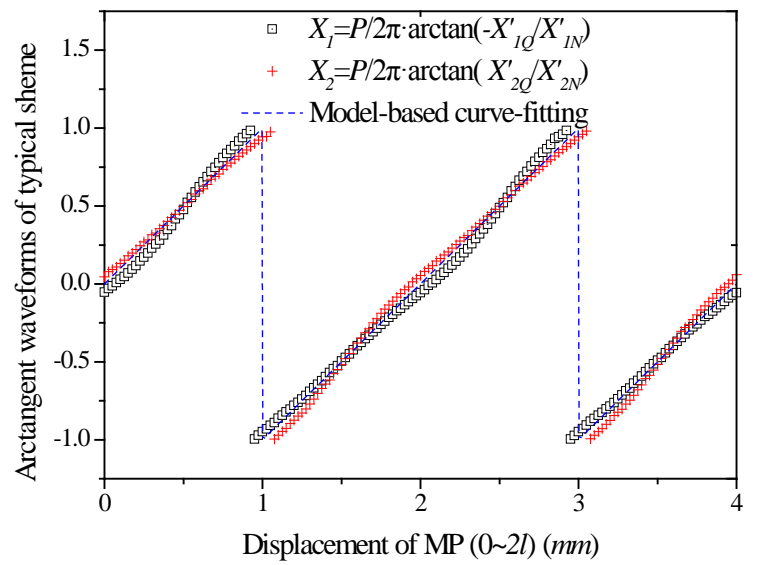

Fig.7. Arctangent waveforms directly from normalized waveforms under measurement disturbances. 
The decoupling method in (14) and (15) removes the influences of sensitivity differences and phase-shift errors, theoretically. Fig.9. shows the acquired arctangent waveform from (14), with displacement measurement in $X$ direction as an example. The waveform from (14) is more linear than the waveforms in Fig.7. After applying PSA interpolation method when $N=8,16$ pulses are generated in a full period. Calculating the displacement information in the last pulse ensures that fine displacement can be achieved from (16).

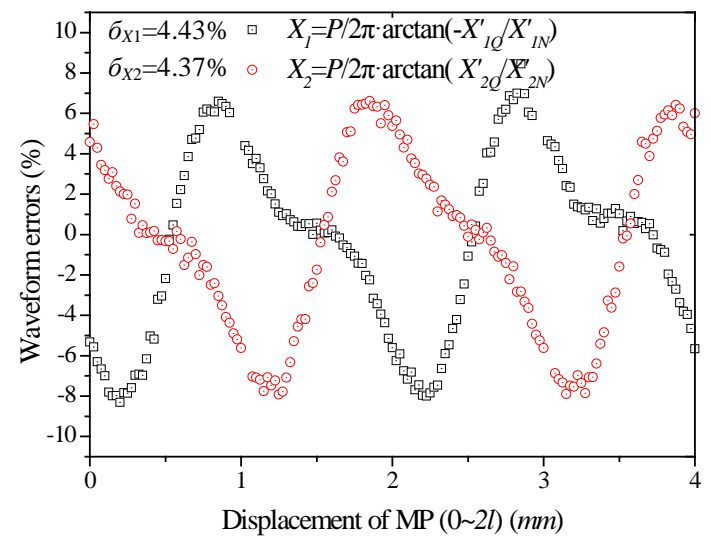

Fig.8. Waveform errors of arctangent signals directly from normalized waveforms under measurement disturbances.

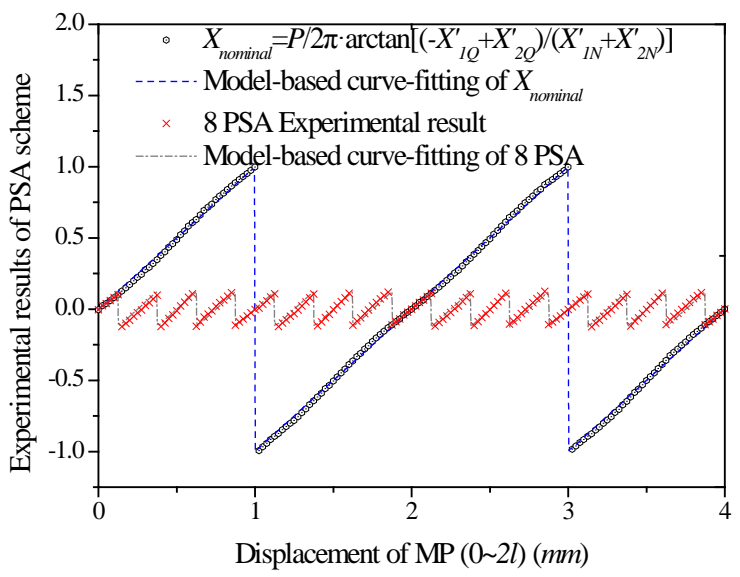

Fig.9. PSA waveform when $N=8$.

Fig.10. depicts the waveform errors of the measured PSA waveform in a single period. Results show that the PSA waveform has less than $3 \%$ nonlinearity errors to arctangent waveforms, and the standard deviation is as low as $1.72 \%$. PCIDS signals are not actually in perfect sinusoidal or cosine model. Thus, $1.72 \%$ waveform error remains. Otherwise, the PSA method can still improve signal linearity. Specific causes of this error include parasitic capacitance, humidity, temperature, and thickness of capacitor electrodes. These results indicate that the effects of static errors and dynamic disturbances have been mostly removed. Thus, the PSA displacement interpolation method in Sec. 3 ensures the satisfactory performance of PCIDS even under measurement disturbances.

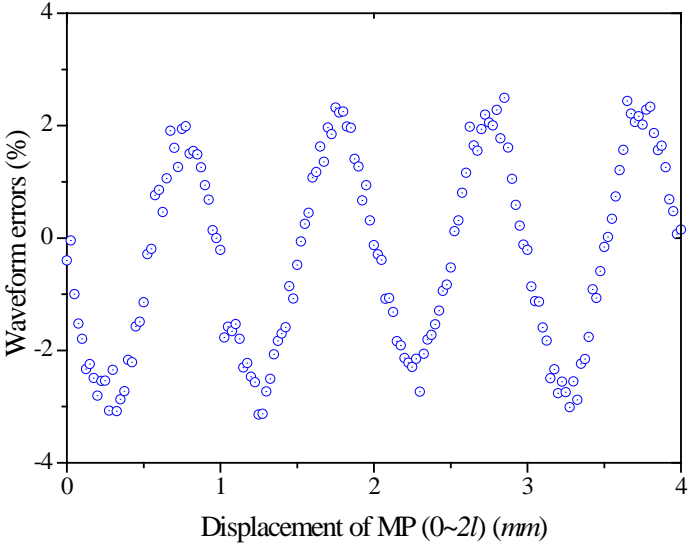

Fig.10. Waveform errors of $X_{\text {nominal }}$ from PSA interpolation method under measurement disturbances.

\section{CONCLUSION}

This paper proposes a novel PSA interpolation method to reduce the influences of static errors and dynamic disturbances on PCIDS accurate measurement. On the basis of the acquired PCIDS capacitance model against static and dynamic disturbances, PSA interpolation method first removes the phase-shift errors and sensitivity differences by applying a particular arctangent function. Measurement resolution is then improved by combining coarse measurement and fine estimation. Experiments show that waveform errors decreased significantly from more than $4 \%$ to $1.72 \%$ after applying the PSA interpolation method. Advantages of the PSA interpolation method are validated.

\section{ACKNOWLEDGMENT}

The authors would like to thank the financial supports by National Natural Science Foundation of China (Grant No. 51405263, 51275272, 51275465).

\section{REFERENCES}

[1] Barker, M.J., Colclough, M.S. (1997). A two-dimensional capacitive position transducer with rotation output. Review of Scientific Instruments, 68 (8), 3238-3240.

[2] Yu, H., Zhang, L., Shen, M. (2015). Novel capacitive displacement sensor based on interlocking stator electrodes with sequential commutating excitation. Sensors and Actuators A - Physical, 230, 94-101.

[3] Ahn, H.J. (2006). A cylindrical capacitive sensor (CCS) for both radial and axial motion measurements. Measurement Science and Technology, 17 (7), 20272034.

[4] Kuijpers, A.A., Krijnen, G.J.M., Wiegerink, R.J., Lammerink, T.S.J., Elwenspoek, M. (2006). A micromachined capacitive incremental position sensor: Part 1. Analysis and simulations. Journal of Micromechanics and Microengineering, 16 (6), S116S124.

[5] Kim, M., Moon, W., Yoon, E., Lee, K.R. (2006). A new capacitive displacement sensor with high accuracy and long-range. Sensors and Actuators A Physical, 130, 135-141. 
[6] Huang, X.H., Lee, J.I., Ramakrishnan, N., Bedillion, M., Chu, P. (2010). Nano-positioning of an electromagnetic scanner with a MEMS capacitive sensor. Mechatronics, 20 (1), 27-34.

[7] Benedek, P., Silvester, P. (1972). Capacitance of parallel rectangular plates separated by a dielectric sheet. IEEE Transactions on Microwave Theory and Techniques, MTT-20 (8), 504-510.

[8] Wolff, I., Knoppik, N. (1974). Rectangular and circular microstrip disk capacitors and resonators. IEEE Transactions on Microwave Theory and Techniques, MTT-22 (10), 857-864.

[9] Ahn, H-J., Jeon, S. (2012). Error analysis of a new cylindrical capacitive sensor (CCS) for measuring five-dimensional motions of a rotor. Mechanical Systems and Signal Processing, 29, 148-163.

[10] Heydemann, P.L.M. (1981). Determination and correction of quadrature fringe measurement errors in interferometers. Applied Optics, 20 (19), 3382-3384.

[11] Tan, K.K., Zhou, H.X.X., Lee, T.H. (2002). New interpolation method for quadrature encoder signals. IEEE Transactions on Instrumentation and Measurem ent, 51 (5), 1073-1079.
[12] Hu, H.J., Qiu, X.Q., Wang, J., Ju, A.S., Zhang, Y.B. (2009). Subdivision and direction recognition of lambda/16 of orthogonal fringes for nanometric measurement. Applied Optics, 48 (33), 6479-6484.

[13] Benammar, M., Ben-Brahim, L., Alhamadi, M.A., AlNaemi, M. (2008). A novel method for estimating the angle from analog co-sinusoidal quadrature signals. Sensors and Actuators A - Physical, 142 (1), 225-231.

[14] Ryabko, M., Koptyaev, S., Shcherbakov, A., Lantsov, A. (2014). Interferometer-based technology for optical nanoscale inspection. Measurement Science Review, 14 (1), 25-28.

[15] Sysoev, E., Kulikov, R., Vykhristyuk, I., Chugui, Y. (2015). Correction of scanning steps to improve accuracy in interferometric profilometer. Measurement Science Review, 15 (1), 9-12.

[16] Yu, J.P., Wang, W., Lu, K.Q., Mei, D.Q., Chen, Z.C. (2013). A planar capacitive sensor for 2D long-range displacement measurement. Journal of Zhejiang University SCIENCE C, 14 (4), 252-257.

Received November 3, 2015. Accepted May 27, 2016. 